\title{
Social and motivational functioning is not critically dependent on feedback of autonomic responses: neuropsychological evidence from patients with pure autonomic failure
}

\author{
H. C. Heims ${ }^{\text {a }}$, H. D. Critchley ${ }^{\text {b,c }}$, R. Dolan ${ }^{\text {d }}$, C. J. Mathias ${ }^{\text {b,e }}$, L. Cipolotti ${ }^{\text {f,* }}$ \\ a Department of Neuropsychology, National Hospital for Neurology and Neurosurgery, Box 37, Queen Square, London WC1N 3BG, UK \\ ${ }^{\mathrm{b}}$ Autonomic Unit, National Hospital for Neurology and Neurosurgery, Queen Square, London WC1N 3BG, UK \\ ${ }^{\mathrm{c}}$ Institute of Cognitive Neuroscience, UCL, UK \\ ${ }^{\mathrm{d}}$ Wellcome Department of Imaging Neuroscience, Institute of Neurology, UCL, UK

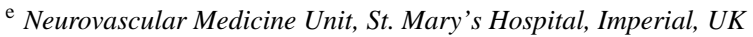 \\ ${ }^{\mathrm{f}}$ Dipartimento Di Psicologia, University of Palermo, Italy
}

Received 27 January 2004; accepted 18 May 2004

\begin{abstract}
Social, emotional and motivational behaviours are associated with production of automatic bodily responses. Re-representation in the brain through feedback of autonomic and skeletomuscular arousal is proposed to underlie "feeling states". These influence emotional judgments and bias motivational decision-making and guide social interactions. Consistent with this hypothesis, dissocial behaviour and deficits on emotional and motivation tasks are associated with blunted bodily responses in patients with orbitofrontal brain lesions or developmental psychopathy. To determine the critical dependence of social and emotional behaviours on bodily responses mediated by the autonomic nervous system, we examined patients with pure autonomic failure (PAF), a peripheral denervation of autonomic neurons with onset in middle age.

Compared to healthy subjects, PAF patients were unimpaired on tests of motivational decision-making (Iowa Gambling Task), recognition of emotional facial expressions, Theory of Mind Tasks and tests of social cognition. Only on a test of emotional attribution, which is perhaps more sensitive to subjective feeling states, did PAF patients score worse than the comparison group, though there was no evidence that this deficit was specific to a discrete emotion and requires further validation.

These findings suggest that emotional and social functioning is not critically tied to on-going experience of autonomic arousal state, Acquisition of autonomic failure late in life may protect against maladaptive social behaviour through established behavioural responses that may be associated with central "as if" representations.
\end{abstract}

(c) 2004 Elsevier Ltd. All rights reserved.

Keywords: Pure autonomic failure; Motivational decision-making; Social behaviour

\section{Introduction}

Bodily states of arousal, mediated in part through the action of the autonomic nervous system, are proposed to play an important role in emotional, motivational and social behaviour (Damasio, Tranel, \& Damasio, 1991; Damasio, 1994, 1999). Whereas outward expressions of autonomic activity such as sweating, and facial flushing may act as social cues, awareness of internal bodily responses underlie feeling states and "gut feelings" that may colour or intensify

\footnotetext{
* Corresponding author. Tel.:+44-20-7829-8793; fax: +44-20-7813-2516.

E-mail address: 1.cipolotti@ion.ucl.ac.uk (L. Cipolotti)
}

emotional experience, motivate behaviour and guide social interactions (James, 1894; Lange, 1922; Damasio, 1999).

Visceral and skeletomotor responses have long been recognized as integral components of emotional expressions, conserved evolutionarily across animal species (Darwin, 1998). The close relationship between bodily state and emotion is central to the James-Lange theory of emotion, which argues that the essence of affective experience is the brain's perception of bodily arousal state without which there could be no emotion (James, 1894). Visceral and cardiovascular responses, controlled by the autonomic nervous system provided the obvious substrate mediating "emotional" bodily reactions. However, because autonomic arousal may be unspecific compared to the range of different emotional states, an alternative argument was proposed that changes in bodily 
arousal accompany, but is not a critical component of, subjective emotional experience (Cannon, 1927). Nevertheless, autonomic arousal may non-specifically enhance emotional intensity and provide a basis for cognitive appraisal of the type of emotion to be experienced (Schachter \& Singer, 1962). More recent evidence indicates bodily arousal states can implicit bias to motivational or emotional behaviour (Damasio, 1994; Katkin, Weins, \& Ohman, 2001) and enhance memory consolidation (Cahill \& McGaugh, 1998).

The Somatic Marker Hypothesis, proposed by Damasio and co-workers, embodies many of the concepts of the James-Lange Theory of Emotion (Damasio et al., 1991; Damasio, 1994, 1999). Central mapping of automatically elicited bodily responses ('somatic markers') are proposed to guide social, emotional and motivational behaviour. This hypothesis arose from observations in patients with orbitofrontal cortex damage, who may score normally on standard neuropsychometric testing (including tests of executive function) yet exhibit marked social emotional and motivational dysfunction in daily life (Damasio et al., 1991; Damasio, 1994; Bechara, Tranel, Damasio, \& Damasio, 1996; Bechara, Damasio, Damasio, \& Lee, 1999). These patients exhibit deficits in motivational decision-making, demonstrated using the Iowa Gambling Task (Bechara et al., 1996). In this task, subjects continuously select cards from a choice of four decks, where each card indicates the amount of money won or lost on that trial. Selecting cards from two of the decks results in high immediate reward or (very) high immediate punishment and, if repeatedly chosen, produces an overall loss of money. Selecting cards from the other two decks results in low immediate reward or punishment but, if played repeatedly, achieves in an overall monetary gain. Healthy subjects learn to adapt their selection in favour of the more advantageous low-risk options over the course of the experiment and develop anticipatory arousal responses (measured electrodermally) when contemplating risky decisions even before they are aware of the actual risk (Bechara et al., 1996, 1999; Bechara, Damasio, Tranel, \& Damasio, 1997). Patients with orbitofrontal damage do not develop these implicit arousal responses to contemplation of risk, and do not learn to adapt their decisions advantageously (yet may accurately describe which decks are associated with monetary loss) (Bechara et al., 1996, 1997, 1999). The Somatic Marker Hypothesis proposes that automatically generated bodily arousal responses feed back to guide motivational behaviour, predicting that absent bodily arousal responses will be associated with behavioural deficits (Damasio et al., 1991; Damasio, 1994; Bechara et al., 1996, 1997, 1999; Bechara, Damasio, \& Damasio, 2003; Tranel, 2000) and perhaps influence emotional processing (Damasio, 1994; Blair \& Cipolotti, 2000) and social cognition (Damasio, 1994; Blair \& Cipolotti, 2000).

Emotional processing and empathetic responses may depend on representations of bodily reactions and arousal states. Autonomic (electrodermal) arousal responses are elicited in healthy subjects viewing emotional facial ex- pressions or emotive pictures of either positive or negative valence emotion (Katkin et al., 2001; Blair \& Cipolotti, 2000; Herpertz et al., 2001). Abnormal autonomic responses to facial emotion and deficits in recognition of expressions (particularly anger) occur together in patients with acquired sociopathy following orbitofrontal damage (Blair \& Cipolotti, 2000). Psychopathic individuals (developmental sociopathy) also show blunted autonomic arousal to emotional material, especially sad and fearful expressions, and show recognition deficits for these emotions (Herpertz et al., 2001; Aniskiewicz, 1979; Blair, Jones, Clark, \& Smith, 1997; Blair, Colledge, \& Mitchell, 2001a). Moreover, social cognition, i.e. knowledge about the social appropriateness of actions and inference regarding the thoughts of others (mentalizing abilities, Theory of Mind), may also be guided by 'empathetic' bodily arousal (Anderson, Bechara, Damasio, Tranel, \& Damasio, 1999). Deficits in social cognition are observed in patients with orbitofrontal cortex or amygdala lesions (Bechara et al., 2003; Blair \& Cipolotti, 2000; Stuss, Gallup, \& Alexander, 2001; Snowdon et al., 2003) and are characteristic of developmental autism (Zahn, Rumsey, \& Van Kammen, 1987; Hirstein, Iversen, \& Ramachandran, 2001). Abnormalities in bodily responses to emotive material are apparent in these patient groups (Bechara et al., 1996, 1999; Tranel, 2000; Blair \& Cipolotti, 2000; Zahn et al., 1987; Hirstein et al., 2001). Together, these findings are consistent with the notion that blunted bodily reactions to emotive material may compromise normal social and emotional processing, contributing to dissocial and maladaptive behavioural repertoires.

Although bodily arousal responses may guide motivational decision-making, emotional processing and social cognition, it is unknown if autonomic responses (such as electrodermal activity) represent the principal avenue of bodily feed back for behavioural guidance. Bodily arousal states are also manifest in (non-autonomic) skeletomotor responses such as eyeblink, startle, muscle activity, tension and joint position, independently of autonomic nervous control. Even patients with complete cervical spinal transection retain a dynamic representation of sensorimotor bodily responses of the head and face (Damasio, 1994). To determine if motivational decision-making, emotion processing and social cognition are indeed dependent on autonomic responses, we examined these functions in patients who cannot generate (or feed back) integrated peripheral autonomic responses but who have intact somatomotor responses. We therefore examined patients with pure autonomic failure (PAF). PAF is a disorder of unknown aetiology beginning in middle age that results in peripheral postganglionic degeneration of sympathetic and parasympathetic autonomic neurons, with no evidence of central neuronal degeneration (Mathias, 2000; Mathias \& Bannister, 1999). PAF results in an inability to modulate bodily state via the autonomic nervous system. Consequently, there is no integrated central feedback of autonomic changes that normally accompany behaviour (Mathias, 2000; Mathias \& 
Bannister, 1999; Critchley, Mathias, \& Dolan, 2001). Patients with PAF do not generate increases in heart rate or blood pressure during emotional processing or mental and physical stress (Mathias \& Bannister, 1999; Critchley et al., 2001; Mathias, Mallipeddi, \& Bleasdale-Barr, 1999), nor do they increase circulatory catecholamines during emotion challenge (Mathias, 2000; Mathias \& Bannister, 1999). Furthermore, they have absent skin conductance responses to emotive and orienting stimuli (Magnifico, Misra, Murray, \& Mathias, 1998). By examining performance of PAF patients on tests of motivational decision-making, emotional processing and social cognition, we sought to determine the extent to which on-going autonomic bodily responses underpin these aspects of adaptive behaviour.

In summary, clinical studies link abnormal peripheral arousal responses, reflected in abnormal autonomic activity, with deficits in motivational decision-making, recognition deficits in processing emotional facial expressions and behavioural and psychological indices of impairments in social cognition (Bechara et al., 1996, 1997, 1999; Blair \& Cipolotti, 2000; Herpertz et al., 2001; Aniskiewicz, 1979; Blair et al., 1997; Zahn et al., 1987; Hirstein et al., 2001). Influential theories of emotion and cognition emphasize the importance of bodily arousal to the control of social, emotional and motivational behaviour (Damasio et al., 1991; Damasio, 1994, 1999; James, 1894; Lange, 1922; Schachter \& Singer, 1962). A key question is how crucial autonomic arousal responses, relative to other peripheral (e.g. skeletomotor) arousal responses, are to these psychological processes dependent on somatic arousal. The aim of this study was to address the question as to whether the absence of peripheral autonomic responses in PAF patients will impair performance of tasks sensitive to motivational decision-making, emotion processing and social cognition.

\section{Methods}

\subsection{Patients}

Six patients with a clinical diagnosis of pure autonomic failure participated in the study. They were all recruited from the Autonomic Unit of The National Hospital For Neurology and Neurosurgery. They were aged between 58 and 82 years (mean age $=70.83$; S.D. $=9.15$ ). Five were female and one was male. All six patients were referred to the Neuropsychology Department of the National Hospital for Neurology and Neurosurgery, for evaluation of their cognitive functions. Details of their neuropsychological evaluation are reported in Table 1. Overall the six patients' performance on a series of cognitive tasks tapping intellectual functioning, memory, naming, literacy and calculation, perception, 'executive' and speed and attention functions was well preserved. Only very subtle and varied cognitive deficits were present. We also evaluated the patients' face perception abilities since in the experimental investigation (see below) we administered a task requiring identifying emotional facial expression. Two tests were administered: the famous faces identification test (Warrington \& James, 1967) and the face-matching test (see for details Cipolotti, Robinson, Blair, \& Frith, 1999). In the famous faces identification test the patients' performance was contrasted with that of 39 elderly normal controls (mean age 62 years; range 55-70). In the face matching test the patients' performance was contrasted with that of 30 younger controls (see Cipolotti et al., 1999). All patients performed satisfactorily on both tasks demonstrating preserved face processing skills (see Table 2).

\subsection{Comparison group}

In the experimental investigation the performance of patients with PAF was compared with a group of 18 healthy volunteer subjects, matched for age, who were free from neurological impairment. They were aged between 50 and 82 years $($ mean age $=66.61$; S.D. $=12.91$ ). Eleven were female and 7 were male.

\subsection{Experimental investigation}

All six patients were administered a series of tests designed to investigate decision-making, emotional expression recognition and social cognition. The only exception is represented by the emotional expression recognition, which could be administered only to five patients.

\subsection{Motivational decision-making}

The computerised version of the Iowa Gambling Task was administered (Bechara, Damasio, \& Damasio, 2000). This is a gambling task that stimulates real-life decision-making. Participants are presented with four decks of cards. Decks A \& B result in high reward and punishment, and if played continuously, result in net loss. Decks C \& D result in low reward and punishment, but result in a net profit, if they are played continuously. Participants must learn to avoid the net loss packs (A \& B) in favour of the net gain packs $(C \& D)$. The number of cards selected from decks A \& B and C \& $\mathrm{D}$ are counted in blocks of 20 cards, resulting in a total of 100 cards. A net score is derived for each block $(\mathrm{C}+\mathrm{D})-$ $(\mathrm{A}+\mathrm{B})$; negative scores reflect disadvantageous selection, positive scores, advantageous selection.

\subsection{Emotional expression recognition}

A variation of the emotional expression multimorph task was administered (Frigerio, Burt, Montagne, Murray, \& Perrett, 2002; Coupland, Singh, Sustrik, Ting, \& Blair, 2003). This task contains examples of six basic emotional facial expressions (happiness, surprise, fear, sadness, disgust and anger), taken from the validated Pictures of Facial Affect Series (Ekman \& Friesen, 1976). It consists of a continua composed of photographic pictures of faces, with 
Table 1

Cognitive test scores

\begin{tabular}{|c|c|c|c|c|c|c|c|c|c|c|c|c|}
\hline \multirow[t]{2}{*}{ Test } & \multicolumn{2}{|c|}{ Patient 1} & \multicolumn{2}{|c|}{ Patient 2} & \multicolumn{2}{|c|}{ Patient 3} & \multicolumn{2}{|c|}{ Patient 4} & \multicolumn{2}{|c|}{ Patient 5} & \multicolumn{2}{|c|}{ Patient 6} \\
\hline & Raw & Percentile & Raw & Percentile & Raw & Percentile & Raw & Percentile & Raw & Percentile & Raw & Percentile \\
\hline VIQ & 99 & & 110 & & 112 & & 113 & & 106 & & 94 & \\
\hline PIQ & 94 & & 117 & & 102 & & 124 & & 115 & & 104 & \\
\hline NART IQ & 97 & & 124 & & 112 & & 113 & & 122 & & 107 & \\
\hline RMT-W & $24 / 25$ & 50 & $50 / 50$ & $>95$ & $25 / 25$ & 90 & $46 / 50$ & 75 & $44 / 50$ & $50-75$ & $44 / 50$ & $50-75$ \\
\hline RMT-F & $21 / 25$ & 25 & $40 / 50$ & $25-50$ & $18 / 25$ & $5-10$ & $43 / 50$ & $50-75$ & $46 / 50$ & $>75$ & $47 / 50$ & 95 \\
\hline Story recall (immediate) & 8 & $<$ cut-off & 24 & 25 & 25 & $25-50$ & 20 & $10-25$ & 28 & $25-50$ & 26 & $10-25$ \\
\hline Story recall (delayed) & 12 & $10-25$ & 26 & $25-50$ & 24 & $25-50$ & 14 & $10-25$ & 26 & $25-50$ & 28 & $25-50$ \\
\hline Rey figure (copy) & 17 & $<10$ & 36 & 100 & 16 & $<10$ & 35 & 90 & 35 & 90 & 28 & $<10$ \\
\hline Rey figure (recall) & 3 & $<10$ & 17 & 20 & 3 & $<10$ & 14 & $<10$ & 16 & $10-20$ & 10 & $<10$ \\
\hline GNT/30 & 20 & $50-75$ & 28 & $>95$ & 13 & 5 & 26 & 95 & 28 & $>95$ & 18 & $25-50$ \\
\hline GDST/30 & 12 & 15 & 26 & 90 & 23 & 70 & 24 & $>75$ & 23 & $50-75$ & 11 & $10-25$ \\
\hline GDAT/24 & 6 & $10-25$ & 20 & $>75$ & 19 & $>75$ & 17 & $>75$ & 6 & $10-25$ & 1 & $<1$ \\
\hline Silhouettes/30 & 21 & $25-50$ & 23 & $>50$ & 17 & $5-25$ & 19 & 25 & 25 & $>50$ & nt & nt \\
\hline Cube analysis/10 & 9 & $>5 \%$ cut-off & 10 & $>5 \%$ cut-off & 9 & $>5 \%$ cut-off & 10 & $>5 \%$ cut-off & 9 & $>5 \%$ cut-off & 7 & $>5 \%$ cut-off \\
\hline Cancel 0s (s) & 60 & & 43 & $>50$ & 80 & & 59 & & 41 & $>50$ & 74 & \\
\hline Hayling Scs & 4 & low av. & 6 & av. & 3 & poor & 5 & modav & 6 & av. & 6 & av. \\
\hline WCST/6 & 2 & & 6 & & nt & & 6 & & 6 & & 6 & \\
\hline Stroop-C/112 & 112 & 100 & 112 & 100 & 112 & 100 & 112 & 100 & 112 & 100 & 112 & 100 \\
\hline Stroop-CW/112 & 31 & $<1$ & 112 & $100 \%$ & 50 & $<4$ & 96 & 44 & 91 & 36 & 80 & $20-24$ \\
\hline TMA & 48 & $50-75$ & 36 & $75-90$ & 58 & $25-50$ & 38 & $50-75$ & 48 & 50 & 40 & $25-50$ \\
\hline TMB & 115 & $50-75$ & 56 & $>90$ & 112 & $50-75$ & 93 & $50-75$ & 97 & $50-75$ & disc & $<1$ \\
\hline FAS & 20 & $10-20$ & 69 & $>90$ & 26 & $30-40$ & 36 & $50-60$ & 55 & $>90$ & nt & nt \\
\hline Animals & 11 & 10 & 26 & $>90$ & 13 & $25-50$ & 14 & 25 & 20 & $75-90$ & 19 & $25-50$ \\
\hline
\end{tabular}

Key: VIQ: verbal IQ (Wechsler, 1981); PIQ: performance IQ (Wechsler, 1981); NART: national adult reading test (Nelson, 1982); RMT-W: recognition memory test-words (Warrington, 1984); RMT-F: recognition memory test-faces (Warrington, 1984);Story recall (Coughlan \& Hollows, 1985);Rey figure (Rey, 1964); GNT: graded naming test (McKenna \& Warrington, 1980); GDST: graded difficulty spelling test (Baxter \& Warrington, 1994); GDAT: graded difficulty arithmetic test (Jackson \& Warrington, 1986); Silhouettes (Warrington \& James, 1991); Cube analysis (Warrington \& James, 1991); Cancel 0s (Willison \& Warrington, 1992); (Hayling Scs: scaled score (Burgess \& Shallice, 1997); WCST: Wisconsin card sorting test (Nelson, 1976); Stroop C: colour; CW: colour-word (Trenerry, Crossen, DeBoe, \& Leber, 1989); TMA: trail making test A (Army Individual Test Battery, 1944); TMB: trail making test B (Army Individual Test Battery, 1944); FAS: word fluency (Spreen \& Strauss, 1998); Animals: category fluency (Spreen \& Strauss, 1998); nt: not tested; disc: discontinued.

21 morphed images for each continuum. Eighteen stimuli were prepared by blending a prototypical expression (100\% expression) in varying proportions with a neutral expression ( $0 \%$ expression). The neutral face is gradually morphed through 20 stages in 5\% increments into one of the six prototypical expressions. Each face was presented on a computer screen.

Participants viewed each face change rapidly from a neutral to a prototypical expression. They were first asked to state the emotion expressed from a choice of six emotions (happy, sad, anger, disgust, fear, surprise). Subsequently, the same face changed slowly from neutral to the emotion previously displayed. Subjects were required to state at which stage they recognized the emotion by pressing a key on the computer keypad. Following a practice phase consisting of one example of each emotion, the 18 test stimuli were presented in a random order. The task was performed twice, thus for each emotion, there were six presentations. This task was scored according to published procedures (Coupland et al., 2003; Blair, Colledge, Murral, \& Mitchell, 2001b). The number of stages required before successful expression recognition took place was recorded automatically in an excel spreadsheet on the computer. These stages were then converted into a score between 1 and 20. One point was scored for successful recognition of the prototypical expression (stage 20). Twenty points were scored for successfully recognizing a 5\% morph (stage 1), as it is 19 stages away from the prototype. Eleven points were scored

Table 2

Face processing tests: summary of scores

\begin{tabular}{lcccccccc}
\hline Test & Patient 1 & Patient 2 & Patient 3 & Patient 4 & Patient 5 & Patient 6 & Healthy volunteers \\
\cline { 3 - 8 } & & & & & & Range & $38-100$ cum freq & 39 \\
\hline FF/12 & 10 & 12 & 11 & 10 & 10 & 9 & 30 \\
Face matching/12 & 9 & 11 & 11 & 11 & 11 & 11 & $9-12$ \\
\hline
\end{tabular}

Key: FF: famous faces test (Warrington \& James, 1967); cum freq: cumulative frequency; $N$ : number of healthy volunteers. 
for successfully recognizing a 50\% morph (stage 10), as it is 10 stages away from the prototype. Incorrect recognition scored 0 .

\subsection{Social cognition}

A modified battery of the social cognition tasks described by Blair and Cipolotti (2000) was administered. This battery addressed several aspects of social cognition.

\subsubsection{The emotion attribution task}

This task assessed ability to represent the emotions of others. In this task, the participant was presented with 75 short stories describing an emotional situation and was required to provide an emotion describing how the main character might feel in that situation. The sentences were designed to elicit attributions of happiness, sadness, fear, anger or embarrassment. There were 15 sentences for each emotion. The task was scored according to the number of correct attributions made for each emotion category, thus for each emotion, participants received a score out of 15 .

\subsubsection{Theory of Mind task}

This task assessed ability to represent the internal mental state of others. The participant was presented with 15 stories describing naturalistic social situations and was asked to interpret and justify the behaviour of the main character. Three scores were manually recorded. The first indexed comprehension of the situation (maximum score $=15$ ). The remaining two scores referred to the justification provided during interpretation of the story character's behaviour, i.e. reference to either the character's mental states or physical information. Of the stories correctly understood, total number of stories justified using mental state information and total number of stories justified with reference to physical information were recorded.

\subsubsection{The social situations task}

This task assessed ability to process the appropriateness of behaviour in different social contexts. Twenty short stories describing social situations incorporating behaviour that was either normative or a violation were read by the participant. Participants were required to judge the appropriateness of behaviours at various points in each story, giving a score from A to D. A indicated that the behaviour had been judged as normative. Scores of B-D indicated that the behaviour had been judged as a violation and indicated the extent of the violation ( $\mathrm{B}=$ mild, $\mathrm{D}=$ serious). In total, there were 17 normative situations and 20 violations. Three scores were generated. The first two indicate the number of normative situations (maximum 17) and the number of violations (maximum 20) correctly identified. The last score indicates the extent to which participants judged violations to be socially inappropriate. For each violation, the participant obtained a score between 0 and 3, matching their response of $A-D$ (i.e. $A=0, B=1, C=2, D=3$ ).
Table 3

Performance on the Iowa Gambling Task

\begin{tabular}{lll}
\hline \multirow{2}{*}{ Block } & \multicolumn{2}{l}{ Mean net score (S.D.) } \\
\cline { 2 - 3 } & PAF patient group $(n=6)$ & Comparison group $(n=18)$ \\
\hline $1-20$ & $-0.67(3.93)$ & $-3.19(4.61)$ \\
$21-40$ & $-3.00(9.19)$ & $-1.38(4.77)$ \\
$41-60$ & $+10.67(9.00)$ & $+2.25(8.32)$ \\
$61-80$ & $+12.33(8.43)$ & $+3.50(7.92)$ \\
$81-100$ & $+14.00(9.30)$ & $+3.38(10.53)$ \\
\hline
\end{tabular}

\subsection{Statistical analysis}

Univariate ANOVAs were performed on the data obtained for the Iowa Gambling Task, the emotional expression multimorph task and the emotional attribution task. Independent sample $t$-tests were used in order to compare mean scores between the two groups on both the Theory of Mind and social situations tasks. Differences were considered significant at the $P<0.05$ level.

\section{Results}

\subsection{Motivational decision-making}

Table 3 and Fig. 1 present the performance of the PAF patient group with the comparison group on the Iowa Gambling Task. The PAF patients shifted their preference towards the advantageous decks (C \& D) and away from the disadvantageous decks (A \& B) in a similar manner to the control group. This is reflected by the shift in the net scores towards positive. Interestingly, the patient group performed significantly better than the control group on this task. A univariate ANOVA of the net scores on group and emotion revealed a significant main effect of group $(F(1,100)=12.054, P<$

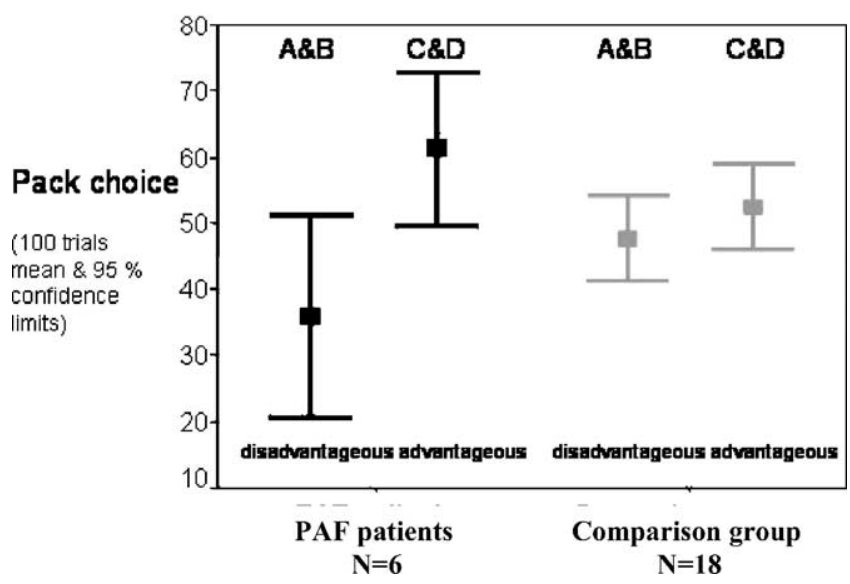

Fig. 1. Iowa Gambling Task. The average performance of patients with pure autonomic failure (PAF) and a healthy matched comparison group is plotted, showing percentage selection from disadvantageous (A \& B) and advantageous packs ( $\mathrm{C} \& \mathrm{D})$. PAF patients overall demonstrated a more adaptive pattern of responding than the comparison group. Mean and $95 \%$ confidence limits are plotted against trials. 
Table 4

Performance on the emotional expression multimorph task

\begin{tabular}{lll}
\hline Emotion & \multicolumn{2}{l}{ Mean expression recognition } \\
\cline { 2 - 3 } & PAF patient group $(n=5)$ & Comparison group $(n=18)$ \\
\hline Happy & $9.89(3.89)$ & $8.56(3.08)$ \\
Surprised & $7.45(4.62)$ & $5.97(2.86)$ \\
Disgusted & $7.76(4.67)$ & $6.33(2.48)$ \\
Angry & $6.60(4.71)$ & $5.76(3.34)$ \\
Sad & $7.37(3.46)$ & $5.83(2.93)$ \\
Fearful & $7.88(3.37)$ & $5.58(3.36)$ \\
\hline
\end{tabular}

0.001). Indeed, the patient group made significantly more advantageous choices than the control group. The ANOVA also revealed a significant main effect of block $(F(4,100)$ $=8.486, P<0.001)$. There was no interaction of group with block $(F(4,100)=1.915$, n.s. $)$. These findings suggest that, in subjects above the age of 50 years, performance of the Iowa Gambling Task, a test of motivational learning from feedback that requires decision-making for prospective gain to override effects of short term rewards, is not critically dependent on feedback of bodily responses mediated via the autonomic nervous system.

\subsection{Emotional expression recognition}

Table 4 reports performance on the emotional expression multimorph task. We report, for each emotion, the mean number of stages that were required before successful expression recognition occurred. A univariate ANOVA of the expression recognition scores on emotion and group revealed a significant main effect of group $(F(1,114)=4.683$, $P<0.05$ ), with PAF patients recognizing the emotional expressions at an earlier stage than the comparison subjects. There was no significant main effect of emotion $(F(5,114)$ $=1.656$, n.s.), nor an interaction between group and emotion $(F(5,114)=0.077$, n.s. $)$.

A second univariate ANOVA was conducted on the error scores. This revealed no main effect of group $(F(1,114)$ $=0.458$, n.s.), indicating that PAF patients made a similar number of recognition errors to the comparison group. Together these findings demonstrate that PAF patients have an intact ability to recognize emotional facial expressions, suggesting that 'empathetic' autonomic arousal responses do not contribute greatly to processing of expressed emotion.

\subsection{Social cognition}

\subsubsection{Emotional attribution task}

Table 5 presents the performance of the patient group with the comparison group on the emotion attribution task. A univariate ANOVA of the number of correct answers revealed a significant main effect of emotion $(F(4,110)=16.763$, $P<0.001)$. Post hoc analyses revealed that both patients and healthy subjects had most difficulty on sentences requiring the attribution of embarrassment $(P<0.001)$. Sim-
Table 5

Mean number of correct answers on the emotional attribution task ${ }^{\mathrm{a}}$

\begin{tabular}{lll}
\hline Emotion & \multicolumn{2}{l}{ Mean number correct (S.D.) } \\
\cline { 2 - 3 } & PAF patient & Comparison \\
& group $(n=6)$ & group $(n=18)$ \\
\hline Happy & $14.83(0.41)$ & $14.89(0.32)$ \\
Sad & $13.33(0.82)$ & $14.11(0.76)$ \\
Fear & $14.00(0.89)$ & $14.28(0.83)$ \\
Anger & $12.33(2.34)$ & $13.83(1.04)$ \\
Embarrassment & $9.67(4.84)$ & $11.78(2.44)$ \\
\hline
\end{tabular}

a The maximum correct score is 15 .

ilarly, both groups found it harder to attribute the emotion of anger to story characters, than the emotion of happiness $(P<0.05)$. There was no interaction of group with emotion $(F(4,110)=1.227$, n.s.). However, the ANOVA did reveal a main effect of group $(F(1,110)=7.468, P<0.01)$, with patients performing worse than the comparison group. The mild underperformance of patients on this task suggests that autonomic bodily responses may play a role in predicting, perhaps through empathetic emulation, the subjective emotional feeling states of others.

\subsubsection{Theory of Mind}

In Fig. 2, the performance of the patient group on the advanced theory of mind task is compared to that of the control group. No significant differences were found between the patients and comparison subjects (tested using Mann-Whitney $U$-tests). There were no significant differences in the number of mental and physical state justifications given by the patients and comparison subjects (mental state: $Z=-0.07$, n.s., physical state: $Z=-1.3$, n.s.). Similarly, there was no significant difference between the PAF and comparison subjects on the number of stories they correctly understood $(Z$ $=1.7$, n.s.). This evidence indicates that PAF patients have an intact ability to judge the internal mental states of others.

\subsection{Social situation task}

Fig. 3 compares the performance of the PAF patient group with the control group on the social situation task. Independent sample Mann-Whitney $U$ analyses revealed no significant differences. The patient group did not significantly differ from the comparison group in their ability to identify normative situations $(Z=-0.34$, n.s.) or violations of normative situations $(Z=-0.14$, n.s.). Moreover, PAF patient scores reflecting the extent of social violations (appropriateness scores) also did not significantly differ from the control group $(Z=-0.23$, n.s.). These findings indicate that the patients have an intact ability to process the appropriateness of behaviour in different social contexts.

\section{Discussion}

Our study provides the first empirical data examining the dependence of social, emotional and motivational function- 


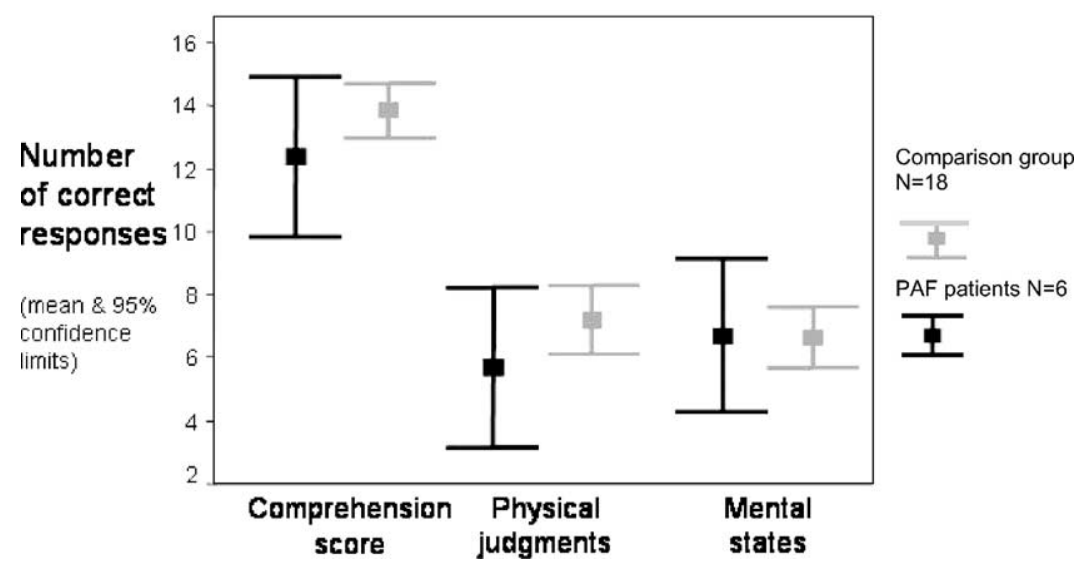

Fig. 2. Theory of Mind Task. Subjects read stories and were assessed on general level of comprehension, understanding of physical explanations for behaviour and of the internal mental state of characters described (i.e. Theory of Mind). Compared to a comparison group, patients with pure autonomic failure showed no significant impairment on this test of social cognition.

ing on the integrity of peripheral autonomic activity. Influential theories of emotion have argued for centrality of cerebral re-representation of automatic bodily responses to emotional feeling states that may bias learning, decision-making and social and emotional processing (Damasio et al., 1991; Damasio, 1994, 1999; James, 1894; Lange, 1922). These theoretical models are corroborated by clinical and psychophysiological evidence that have typically indexed bodily arousal states through involuntary autonomic responses during emotional processing in healthy subjects, patients with focal brain lesions and individuals with developmental deficits in adaptive social and emotional functioning (e.g. Bechara et al., 1996, 1997, 1999, 2003; Tranel, 2000; Blair \& Cipolotti, 2000; Herpertz et al., 2001; Aniskiewicz, 1979; Blair et al., 1997; Zahn et al., 1987; Hirstein et al., 2001). Bodily responses are manifest both through neural modulation of autoregulatory 'visceral' functions (such as sweat gland control) and through non-autonomic reactions of skeletomusculature mediating protective reflexes such as startle and eyeblink and controlling general muscle tone. The present study sought to characterize the unique influence of autonomic responses on processes underlying adaptive social functioning by examining performance of patients with peripheral autonomic denervation on tasks thought sensitive to bodily arousal states.

Our findings broadly indicate that PAF patients have no significant deficits in domains of motivational decisionmaking, expression recognition or social cognition, indicating that these processes do not have obligate reliance on bodily responses mediated autonomically. PAF subjects showed mild underperformance, relative to the comparison group, only in the attribution of appropriate emotional states to characters in scripted scenarios. Previous studies report some blunting of subjective emotional experience in

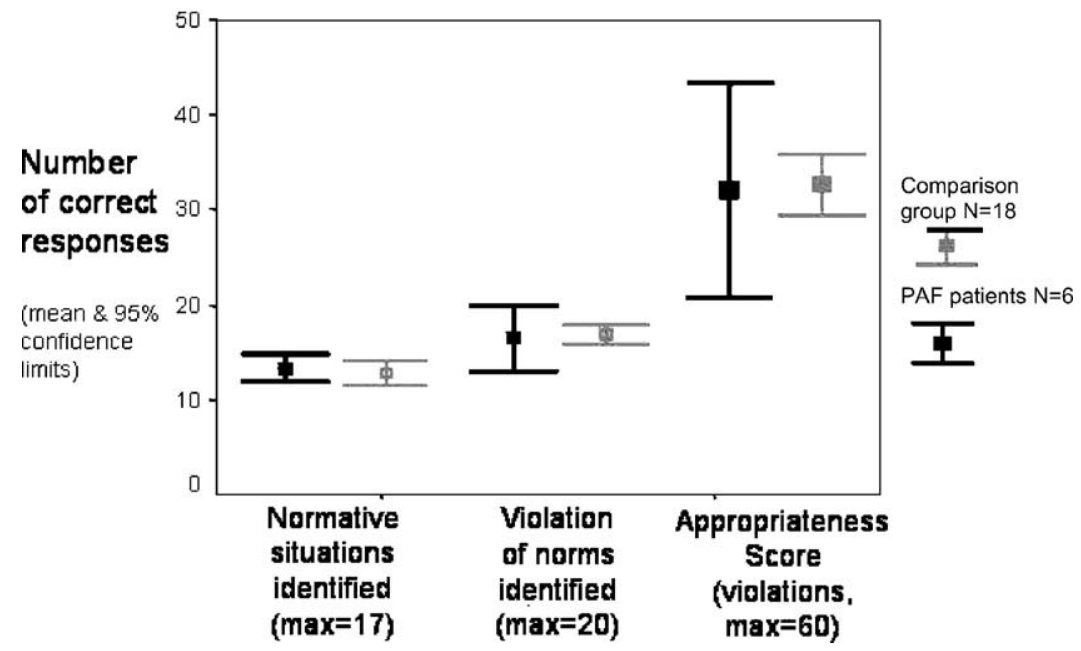

Fig. 3. Social situations task. Subjects were assessed on their ability to process appropriateness of behaviour in different social context from short stories. These stories described social situations incorporating behaviour that was either normative or a violation. Three scores were generated of correctly identified normative situations, violations and the extent to which participants judged violations to be socially inappropriate. Compared to the comparison group, patients with pure autonomic failure showed no significant impairment on this test of social cognition. 
PAF subjects (Critchley et al., 2001), yet there has been no clinical deficits reported in this patient group in everyday social or emotional functioning (Mathias, 2000). This lack of apparent major behavioural confound has made PAF a useful lesion/deficit model for investigation of brain mechanisms related to control of autonomic bodily arousal and the influence of autonomic responses on emotion related neural activity (Critchley et al., 2001; Critchley, Mathias, \& Dolan, 2002). However, this model is confined only to autonomic functions and does not relate to other, non-autonomic, somatic responses. The present study suggests that the absence of integrated autonomic responses may cause subtle impairments restricted to the experience and attribution of feeling states, yet this observation needs further validation in a larger group.

At least two main factors may account for intact performance of PAF subjects on our range of neuropsychological tasks. Firstly, other axes of bodily arousal remain intact (and may even be enhanced) in PAF subjects. Moreover, our subjects were all above the age of 50 , and because symptoms of PAF arise in middle age, consistent replication through experience of emotional and social interactions may lead behavioural repertoires to become independent of bodily arousal states, perhaps through 'as if' representations.

The presence of other avenues mediating bodily arousal in PAF subjects may be one of the critical factors. Psychophysiological studies typically employ a single, non-specific, measure of arousal, such as sympathetic electrodermal activity. When recorded with other responses, such as electromyography, the concept of a generalized arousal, involving both autonomic and non-autonomic systems is broadly confirmed. However, there is some evidence for organ specificity of involuntary bodily responses both within and between autonomic and non-autonomic axes (Ekman, Levenson, \& Friesen, 1983; Sonnby-Borgstrom, 2002; Witvliet \& Vrana, 1995). The absence of an autonomic component to bodily arousal state will not block representations of other, skeletomotor, arousal responses. The dedicated anatomical pathway for conveying information about interoceptive bodily state carries not only visceroceptive information, but also somatosensory sensations such as pain and proprioception (Craig, 2002). This information is conveyed centrally via Lamina I spinothalamic neurons within the spinal cord, merges with vagal afferent information in thalamus and then projects to insula and orbitofrontal cortices where the representation of internal states may provide the basis for feeling states (Craig, 2002). PAF affects peripheral, not central, neurons and hence the pathways and brain structures conveying bodily information and representing feeling states remain intact despite lack of autonomic responses. The 'diffuse' nature of feeling states and the integrity of other non-autonomic 'somatic markers' are likely to account for the normal performance of PAF subjects on tasks such as the Iowa Gambling Task.

A second possible critical factor, that the age of subjects may mitigate against marked deficit in social and motiva- tional processes, is based on the observation that disruption of the 'somatic marker system' results in greater social, emotional and motivational deficits if the disruption occurs during brain development (Blair et al., 2001a,b; Anderson et al., 1999). Experience-dependent association of emotional expressions or social scenarios with bodily arousal states may lead to forward models of bodily responses that can guide behaviour without necessarily requiring production of bodily arousal. Such forward 'as if' models of bodily responses may have a basis in the enhanced activity within structures such as anterior cingulate cortex, when PAF patients perform mental and physical stressor tasks that generate autonomic arousal in healthy subjects (Critchley et al., 2001). While the 'as if' model can account for preserved emotional responses to predictable situations, it does not account well for preserved performance of PAF subjects on the Iowa Gambling Task. This task requires new learning that is putatively guided by somatic markers and therefore cannot be successfully performed from prior knowledge and habitual responses. The role of non-autonomic bodily responses in providing somatic markers may therefore account for the success of PAF subjects in this task. It is also important to note that maladaptive underperformance in the Iowa Gambling Task has been reported in a subsection of the normal elderly population (Denburg, Tranel, Bechara, \& Damasio, 2001) and such individuals may have been over-represented within our comparison sample.

One intriguing observation from this study is the suggestion that the attribution of emotional state (but not other cognitions) may draw on empathetic bodily responses. PAF patients underperformed compared to healthy subjects on the emotional attribution task. However, this was mild and not clearly specific to any particular emotional state. Further research is needed to establish the validity of these findings in a larger patient population. Nevertheless, it is an intriguing notion that, in order to attribute an appropriate emotional state to another, one may need to emulate the same evoked autonomic bodily state. In contrast to the attribution task, PAF subjects did not show deficits in the recognition of emotional facial expressions. This discrepancy may reflect the dominance of automatic, but non-autonomic, mimicry responses of facial muscles when confronted with a facial expression (Sonnby-Borgstrom, 2002; Witvliet \& Vrana, 1995). Furthermore, the attribution task may be more sensitive to deficits in subjective feeling states, in contrast to the (more perceptual) expression recognition task.

In summary, this paper provides the first direct examination of the definitive role of autonomic responses in cognitive processing underlying adaptive social, emotional and motivational behaviour. Our data demonstrate that these cognitive systems are not critically dependent on the autonomic axis controlling bodily arousal states, indicating that feedback of 'somatic marker' may alternatively or primarily draw on somatomotor responses to guide and influence behaviour. 


\section{Acknowledgements}

We are grateful to Professor Bechara for his advice on the Iowa Gambling Task, to Professor Dave Perrett for lending us the emotional expression recognition task and Professor James Blair for invaluable advice on how to interpret this task.

\section{References}

Army Individual Test Battery (1944). Manual \& directions for scoring. Washington, DC: War Department, Adjutant General's Office.

Anderson, S. W., Bechara, A., Damasio, H., Tranel, D., \& Damasio, A. R. (1999). Impairment of social and moral behavior related to early damage in human prefrontal cortex. Nature Neuroscience, 2, 10321037.

Aniskiewicz, A. S. (1979). Autonomic components of vicarious conditioning and psychopathy. Journal of Clinical Psychology, 35, 6067.

Baxter, D. M., \& Warrington, E. K. (1994). Measuring dysgraphia: a graded-difficulty spelling test (GDST). Behavioural Neurology, 7, 107116.

Bechara, A., Tranel, D., Damasio, H., \& Damasio, A. R. (1996). Failure to respond autonomically to anticipated future outcomes following damage to prefrontal cortex. Cerebral Cortex, 6, 215-225.

Bechara, A., Damasio, H., Tranel, D., \& Damasio, A. R. (1997). Deciding advantageously before knowing the advantageous strategy. Science, 275, 1293-1295.

Bechara, A., Damasio, H., Damasio, A. R., \& Lee, G. P. (1999). Differential contributions of the human amygdala and ventromedial pre-frontal cortex to decision making. Journal of Neuroscience, 19, 5473-5481.

Bechara, A., Damasio, H., \& Damasio, A. R. (2000). Emotion, decision making and the orbitofrontal cortex. Cerebral Cortex, 10, 295-307.

Bechara, A., Damasio, H., \& Damasio, A. R. (2003). Role of the amygdala in decision-making. Annals of the New York Academy of Sciences, 985, 356-369.

Blair, R. J., \& Cipolotti, L. (2000). Impaired social response reversal. Brain, 123, 1122-1141.

Blair, R. J., Jones, L., Clark, F., \& Smith, M. (1997). The psychopathic individual: a lack of responsiveness to distress cues? Psychophysiology, 34, 192-198.

Blair, R. J., Colledge, E., \& Mitchell, D. G. (2001a). Somatic markers and response reversal: is there orbitofrontal cortex dysfunction in boys with psychopathic tendencies? Journal of Abnormal Child Psychology, $29,499-511$

Blair, R. J., Colledge, E., Murral, L., \& Mitchell, D. G. (2001b). A selective impairment in the processing of sad and fearful expressions in children with psychopathic tendencies. Journal of Abnormal Child Psychology, 29, 491-498.

Burgess, P. W., \& Shallice, T. (1997). The Hayling \& Brixton tests. Bury St Edmunds, Suffolk: Thames Valley Test Company.

Cahill, L., \& McGaugh, J. L. (1998). Mechanisms of emotional arousal and lasting declarative memory. Trends in Neurosciences, 21, 294-299.

Cannon, W. B. (1927). The James-Lange theory of emotions. American Journal of Psychology, 39, 115-124.

Cipolotti, L., Robinson, G., Blair, J., \& Frith, U. (1999). Fractionation of visual memory: evidence from a case with multiple neurodevelopmental impairments. Neuropsychologia, 37, 455-465.

Coughlan, A. K., \& Hollows, A. K. (1985). In A. K. Coughlan (Ed.), Then adult memory and information processing battery. Leeds: St James' University Hospital.

Coupland, N. J., Singh, A. J., Sustrik, R. A., Ting, P., \& Blair, R. (2003). Effects of diazepam on facial emotion recognition. Journal of Psychiatry Neuroscience, 28, 452-463.
Craig, A. D. (2002). How do you feel? Interoception: the sense of the physiological condition of the body. Nature Reviews Neuroscience, 3, 655-666.

Critchley, H. D., Mathias, C. J., \& Dolan, R. J. (2001). Neural correlates of first and second-order representation of bodily states. Nature Neuroscience, 4, 207-212.

Critchley, H. D., Mathias, C. J., \& Dolan, R. J. (2002). Fear-conditioning in humans: the influence of awareness and arousal on functional neuroanatomy. Neuron, 33, 653-663.

Damasio, A. R. (1994). Descartes' error: emotion, reason and the human brain. New York: Grosset/Putnam.

Damasio, A. R. (1999). The feeling of what happens: body and emotion in the making of consciousness. New York: Harcourt Brace.

Damasio, A. R., Tranel, D., \& Damasio, H. (1991). Somatic markers and the guidance of behaviour. In H. Levin, H. Eisenberg, \& A. Benton (Eds.), Frontal lobe function and dysfunction (pp. 217-228). New York: Oxford University Press.

Darwin, C. (1998). In P. Ekman (Ed.), The expression of the emotions in man and animals (3rd ed.). Oxford University Press.

Denburg, N. L., Tranel, D., Bechara, A., \& Damasio, A. R. (2001). Normal aging may compromise the ability to decide advantageously. Brain and Cognition, 47, 156-159.

Ekman, P., \& Friesen, Q. V. (1976). Pictures of facial affect. Palo Alto: Consulting Psychologists Press.

Ekman, P., Levenson, R. W., \& Friesen, W. V. (1983). Autonomic nervous system activity distinguishes among emotions. Science, 221, 12081210.

Frigerio, E., Burt, D. M., Montagne, B., Murray, L. K., \& Perrett, D. I. (2002). Facial affect perception in alcoholics. Psychiatry Research, 113(1/2), 161-171.

Herpertz, S. C., Werth, U., Lukas, G., Qunaibi, M., Schuerkens, A., Kunert, H. J., Freese, R., Flesch, M., Mueller-Isberner, R., Osterheider, M., \& Sass, H. (2001). Emotion in criminal offenders with psychopathy and borderline personality disorder. Archives of General Psychiatry, $58,737-745$.

Hirstein, W., Iversen, P., \& Ramachandran, V. S. (2001). Autonomic responses of autistic children to people and objects. Proceedings of the Royal Society of London Series B-Biological Sciences, 268, 18831888.

Jackson, M., \& Warrington, E. K. (1986). Arithmetic skills inpatients with unilateral cerebral lesions. Cortex, 22, 610-620.

James, W. (1894). Physical basis of emotion. Psychological Review, 1, 516-529;

James, W.Physical basis of emotion Psychological Review, 101, 205210.

Katkin, E. S., Wiens, S., \& Ohman, A. (2001). Nonconscious fear conditioning, visceral perception, and the development of gut feelings. Psychological Science, 12, 366-370.

Lange, C. (1922). The emotions (I. A. Haupt, Trans.). Baltimore: Williams \& Wilkins (Original work published 1885)

Magnifico, F., Misra, V. P., Murray, N. M., \& Mathias, C. J. (1998). The sympathetic skin response in peripheral autonomic failure-evaluation in pure failure pure cholinergic dysautonomia and dopamine-beta-hydroxylase deficiency. Clinical Autonomic Research, 8, 133-138.

Mathias, C. J. (2000). In W. G. Bradley, R. B. Daroff, G. M. Fenichel, \& C. D. Marsden (Eds.), Neurology in clinical practice (pp. 2131-2165). Woburn, MA: Butterworths/Heinemann.

Mathias, C. J., \& Bannister, R. (1999). In C. J. Mathias, \& R. Bannister (Eds.), Autonomic failure: a textbook of clinical disorders of the autonomic nervous system (4th ed., pp. 169-195). Oxford: Oxford University Press.

Mathias, C. J., Mallipeddi, R., \& Bleasdale-Barr, K. (1999). Symptoms associated with orthostatic hypotension in pure autonomic failure and multiple system atrophy. Journal of Neurology, 246, 893-898.

McKenna, P., \& Warrington, E. K. (1983). Graded naming test. Windsor, Berks: NFER-Nelson Publishing Co. Ltd. 
Nelson, H. E. (1976). A modified card sorting test sensitive to frontal lobe defects. Cortex, 12, 313-324.

Nelson, H. E. (1982). The national adult reading test. Windsor, Berks: NFER-Nelson Publishing Co. Ltd.

Rey, A. (1964). L'examen clinique en psychologie (2nd ed.). Paris: Presses universitaires de France.

Schachter, S., \& Singer, J. E. (1962). Cognitive, social and physiological determinants of emotional state. Psychological Review, 69, 379-399.

Snowdon, J. S., Gibbons, Z. C., Blackshaw, A., Doubleday, E., Thompson, J., Craufurd, D., Foster, J., Happe, F., \& Neary, D. (2003). Social cognition in frontotemporal dementia and Huntington's disease. Neuropsychologia, 41, 688-701.

Sonnby-Borgstrom, M. (2002). Automatic mimicry reactions as related to differences in emotional empathy. Scandinavian Journal of Psychology, $43,433-443$.

Spreen, O., \& Strauss, E. (1998). A compendium of neuropsychological tests (2nd ed.). New York: Oxford University Press.

Stuss, D. T., Gallup, G. G., \& Alexander, M. P. (2001). The frontal lobes are necessary for theory of mind. Brain, 124, 279-286.

Tranel, D. (2000). Electrodermal activity in cognitive neuroscience: neuroanatomical and neuropsychological correlates. In R. D. Lane, \& L. Nadel (Eds.), Cognitive neuroscience of emotion (pp. 192-224). New York: Oxford University Press.
Trenerry, M. R., Crossen, B., DeBoe, J., \& Leber, W. R. (1989) Stroop neuropsychological screening test (SNST). Windsor, Berks: NFER-Nelson Publishing Co. Ltd.

Warrington, E. K. (1984). Recognition memory test. Windsor, Berks: NFER-Nelson Publishing Co. Ltd.

Warrington, E. K., \& James, M. (1967). An experimental investigation of facial recognition in patients with unilateral cerebral lesions. Cortex, 3, 317-326.

Warrington, E. K., \& James, M. (1991). The visual object and space perception battery. Bury St Edmunds, Suffolk: Thames Valley Test Company.

Wechsler, D. A. (1981). Wechsler adult intelligence test-revised. London: The Psychological Corporation.

Willison, J. R., \& Warrington, E. K. (1992). Cognitive retardation in a patient with preservation of psychomotor speed. Behavioural Neurology, 5, 113-116.

Witvliet, C. V., \& Vrana, S. R. (1995). Psychophysiological responses as indices of affective dimensions. Psychophysiology, 32, 436443.

Zahn, T. P., Rumsey, J. M., \& Van Kammen, D. P. (1987). Autonomic nervous system activity in autistic, schizophrenic, and normal men: effects of stimulus significance. Journal of Abnormal Psychology, 96, $135-144$ 